DIE ERSTE SEITE

\section{CT-Screening senkt Lungenkrebsmortalität}

Europäische NELSON-Studie

Langjährige starke Raucher profitieren von einem Lungenkrebs-Screening per Lowdose-Volumen-CT: In der NELSON-Studie wurde ein deutlicher Rückgang der durch den Krebs bedingten Mortalität erreicht bei relativ geringer Falsch-positiv-Rate. 13.200 Männer und 2.600 Frauen, allesamt starke Raucher, wurden randomisiert einer
Screening- und einer Kontrollgruppe zugeteilt. Das CT-Screening erfolgte viermal innerhalb von zehn Jahren. Bei den gescreenten Männern war die Lungenkrebssterblichkeit nach zehn Jahren um 24\%, bei den Frauen sogar um 33\% niedriger als in der Kontrollgruppe.

- NEngl J Med 2020; https://doi.org/10.156/NEJMoa1911793

\title{
Erhöhtes Suizidrisiko!
}

\section{Bei kardiologischen Patienten aufs Gemüt achten}

Herzkrankheiten erhöhen die Suizidalität, wie eine retrospektive Kohortenstudie aus Dänemark mit Daten von über 7,2 Millionen Menschen zeigt. Während der Studienperiode von 37 Jahren ereigneten sich 35.483 Suizide. Bei 8.270 Suizidenten war im Vorfeld eine Herzerkrankung diagnostiziert worden. Im Vergleich zur Allgemeinbevölkerung stieg das Suizidrisiko bei
Angina pectoris um 19\%, bei Herzinfarkt um $28 \%$, bei Kardiomyopathie um $41 \%$, bei Vorhofflattern oder -flimmern um $42 \%$, bei Herzinsuffizienz um $48 \%$ und bei ventrikulären Tachykardien um 53\%. Bei Patienten, die einen Herzstillstand überlebt hatten, war es fast fünffach erhöht.

- JIntern Med 2020; https://doi.org/10.1111/JOIM.13025 https://doi.org/10.1111/JOIM.13025

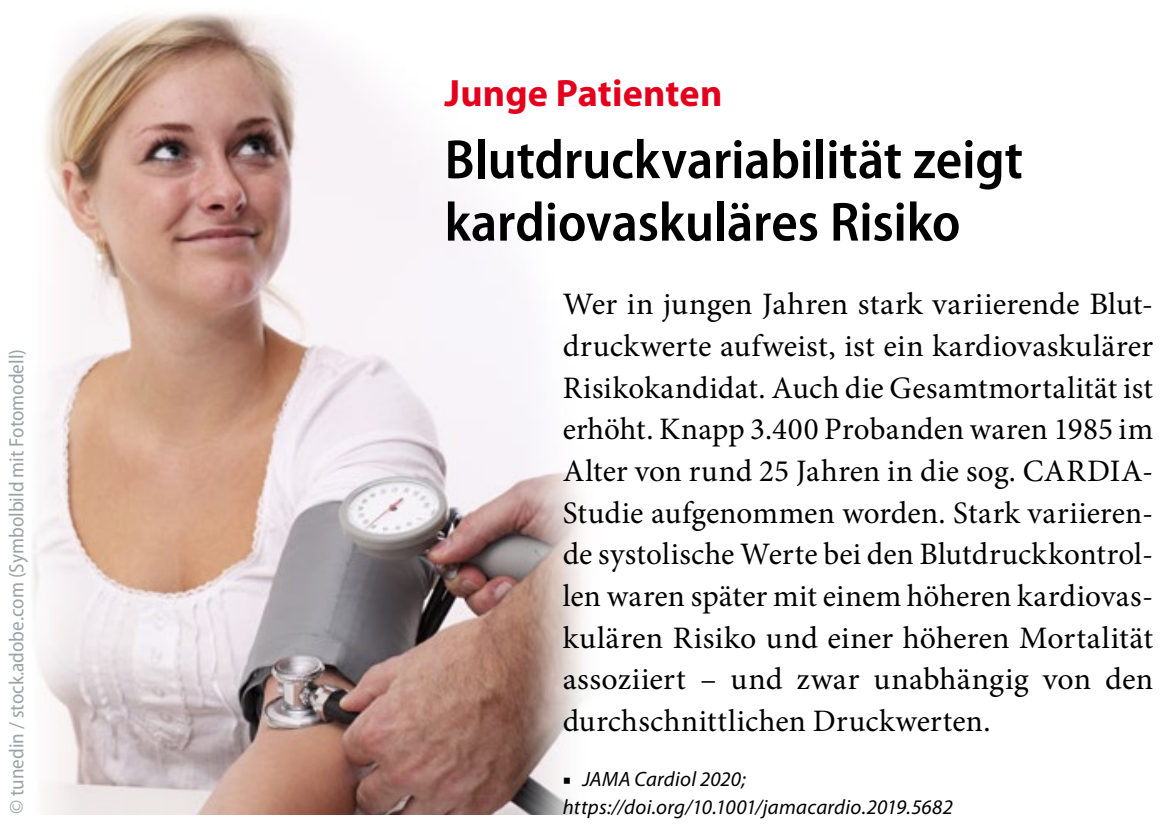

Dr. med. Dirk Einecke

Chefredakteur dirk.einecke@springer.com

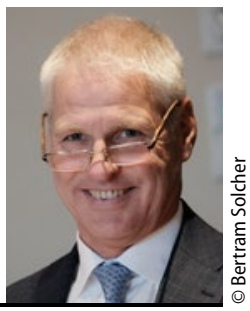

Nach Herzinfarkt

\section{Rezidivgefahr wächst mit dem Bauch}

Einen Indikator für ihre Prognose tragen Postinfarktpatienten gut sichtbar vor sich her. Bei Männern besteht sogar eine lineare Beziehung zwischen abdominaler Adipositas und erneuten kardiovaskulären Ereignissen, unabhängig vom BMI und anderen Risikofaktoren. In die Studie flossen Daten von 23.900 Teilnehmern des

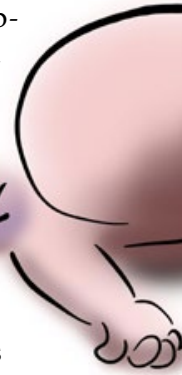
SWEDEHEART-Registers ein.

Fast die Hälfte der Männer und zwei Drittel der Frauen wiesen eine abdominale Adipositas auf. Im Lauf von knapp 4 Jahren kam es bei 7,3\% der Männer und 7,9\% der Frauen zu einem weiteren atherosklerotischen Ereignis. Teilnehmer mit einem Taillenumfang im zweitobersten und obersten Fünftel (Männer $>104$ bzw. $>110 \mathrm{~cm}$, Frauen $>97$ bzw. $>106 \mathrm{~cm}$ ) hatten ein um 21\% bzw. 25\% erhöhtes Risiko verglichen mit Patienten im untersten Fünftel. Bei Männern war der Zusammenhang nahezu linear; bei Frauen lag das geringste Risiko bei einem mittleren Taillenmaß.

- European Journal of Preventive Cardiology 2020; https://doi.org/10.1177/2047487319898019

\section{Coronavirus aktuell}

Das Coronavirus beunruhigt derzeit viele Patienten. MMW-Schriftleiter Prof. Bogner gibt ab S. 8 einen Überblick über den derzeitigen Kenntnisstand. Ein fortlaufend aktualisiertes Dossier zum Thema finden Sie online unter.

www.springermedizin.de/ link/17598658 